\title{
PROGRAMA PREVENTIVO EN SALUD ORAL PARA CONTROLAR FACTORES DE RIESGO ASOCIADOS A CARIES DENTAL Y ENFERMEDAD PERIODONTAL EN MUJERES GESTANTES *
}

\author{
${ }^{1}$ Claudia Milena Riveros Alejo, ${ }^{2}$ Martha Juliana Rodríguez Gómez \\ ${ }^{1}$ Odontóloga U. El Bosque, Especialista en Gerencia Hospitalaria ESAP, \\ Especialista en Relaciones Laborales UNAB, Estudiante II Año Especialización en Odontopediatría U. Santo Tomás. \\ ${ }^{2}$ Odontóloga U. Javeriana, Especialista en Odontopediatría y Ortodoncia Preventiva CES, Docente U. Santo Tomás.
}

Autor responsable de correspondencia: Claudia Milena Riveros Alejo

Dirección de correo electrónico: milevale_odontopediatria@yahoo.es

\section{RESUMEN}

Objetivo: Diseñar, ejecutar y evaluar la efectividad de un programa preventivo en salud oral para controlar los factores de riesgo asociados a caries y enfermedad periodontal en las gestantes afiliadas a una EPS, en Bucaramanga.

Materiales y métodos: Cuarenta gestantes que se encontraban en el segundo y tercer trimestre de embarazo se dividieron, aleatoriamente, en grupo experimental (20) y control (20). A los dos grupos se les suministró un cuestionario sobre creencias en salud oral y prácticas de higiene oral; el estado periodontal, dental y de higiene oral inicial así como las necesidades de tratamiento fueron evaluadas a través de un examen clínico antes y después de un programa preventivo. El grupo experimental recibió terapia mecánica, kits de higiene oral, una conferencia y un video. El grupo control recibió la estrategia educativa desarrollada por la EPS. Para comparar según tipo de intervención se aplicó el test de $X^{2}$ y exacto de Fisher para las variables cualitativas y prueba t de student o test de rangos de Wilcoxon para las cuantitativas.

Resultados: Para el índice de creencias y prácticas saludables sólo se observaron diferencias estadísticamente significativas entre grupo intervenido y control después de la intervención ( $\mathrm{p}<0.0001$ y $\mathrm{p}<0.001$, respectivamente) siendo mejores en el grupo intervenido; igual fenómeno se observó para el índice periodontal comunitario $(\mathrm{p}<0.001)$. Para el índice cuantificado de placa se observaron diferencias estadísticamente significativas entre el grupo intervención y el control antes de la intervención $(\mathrm{p}=0.0038)$, el grupo control reportó mejor nivel de higiene. Sin embargo, después de la intervención la significancia estadística $(\mathrm{p}<0.0001)$ demostró una mejor higiene en el grupo intervenido.

Conclusión: La evidencia parece demostrar que este tipo de programas son eficientes y las EPS pueden implementar estas acciones sin mayor inversión y garantizar la prestación de un servicio integral que se ajuste a las necesidades de esta población. [ Riveros CM, Rodríguez MJ. Programa preventivo en salud oral para controlar factores de riesgo asociados a caries dental y enfermedad periodontal en mujeres gestantes Revista Ustasalud Odontología 2007; 617 - 28]

Palabras Clave: Mujeres gestantes, Enfermedad periodontal, Factores de riesgo, Transmisión de Streptococcus mutans

\section{PREVENTIVE PROGRAM IN ORAL HEALTH TO CONTROL RISK FACTORS ASSOCIATED TO DENTAL CARIES AND PERIODONTAL DISEASE IN PREGNANT WOMEN}

\begin{abstract}
Objective: To design, execute and assess the effectiveness of a preventive program in oral health to control the risk factors for dental decay and periodontal disease in the pregnant women who were affiliated to an "EPS" in Bucaramanga.

Material and methods: Forty pregnant who were in the second and third trimester of pregnancy were divided in experimental (20) and control (20) group. It was provided a questionnaire about the beliefs and practices oral health to both groups. Later, the periodontal index, dental state and oral hygiene index as well as the necessities of treatment were evaluated through a clinical examination before and after a preventive program. The experimental group received mechanical therapy, oral hygiene kits, a conference and a video. The group control received the educative strategy developed by the EPS. In order to compare according by intervention $X^{2}$ and Fisher's exact test were applied by the qualitative variables and $t$ of student or Wilcoxon's rank test were used by the quantitative variables. Results: The significant statistically were seen between intervention and control groups only them of intervention by the beliefs $(p<0.0001)$ and healthful practice index $(p=0.001)$; by both index were seen well in the intervention group. In the same way, ICPTN index was shown associated statistical $(\mathrm{p}<0.001)$. By ICP index statistical association were seen before and after of intervention. Conclusion: The evidence seems to demonstrate that this type of programs are efficient and the EPS could implement this action without greater investment and guarantee the benefit of an integral service that adjusts to the necessities of this population.
\end{abstract}

Key words: Pregnant women, Periodontal disease, Risk factors, Transmission of mutans Streptococci.

Recibido para publicación: 10 de mayo de 2007. Aceptado para publicación: 22 de junio de 2007.

* Grupo de Investigación CYDUSTA. 


\section{INTRODUCCIÓN}

\section{Embarazo y enfermedad periodontal}

A partir del momento de la fecundación, comienzan a producirse en la mujer, una serie de cambios psicológicos, sociales y sistémicos importantes, que normalmente continuarán durante los nueve meses siguientes. Este proceso se conoce como un embarazo. ${ }^{1,2}$

La mujer en su etapa de gestación está expuesta a complejas interacciones hormonales que causan cambios fisiológicos en los sistemas cardiovascular, hematológico, renal, gastrointestinal, endocrino y genitourinario. Los cambios fisiológicos locales ocurren en diferentes partes del cuerpo, incluyendo la cavidad oral. ${ }^{3}$

Se ha demostrado que los síntomas inflamatorios gingivales se agravan durante el embarazo. Durante esta etapa, las hormonas sexuales se desequilibran y afectan algunos órganos. En la encía han sido reconocidos receptores de estrógeno y progesterona lo cual explica, entre otros factores, una mayor respuesta gingival a la placa bacteriana. Por otra parte, la propia composición bacteriana esta modificada debido a la presencia de mayores niveles de progesterona que favorecen el aumento de la Prebotella intermedia. Es común que haya hiperplasia gingival, una mayor irrigación sanguínea y alteración de las fibras presentes en la encía. Esto ocurre frente a un agente agresor, como la placa bacteriana o cálculo dental. ${ }^{4-7}$

En ausencia de una adecuada higiene oral, la gingivitis puede evolucionar a un estado más crítico denominado periodontitis. ${ }^{4}$ La periodontitis se ha definido como la presencia de inflamación gingival en sitios donde ha habido desunión patológica de las fibras de colágeno del cemento, y el epitelio de unión ha migrado apicalmente. Adicionalmente, los eventos inflamatorios asociados con la pérdida de inserción conducen a la resorción de la porción coronal del hueso alveolar de soporte. ${ }^{8-11}$ Los tejidos periodontales inflamados producen importantes cantidades de citoquinas proinflamatorias, principalmente IL-1B, la IL-6, la prostaglandina E2 y factor de necrosis tumoral $\alpha$, los cuales pueden tener efectos sistémicos en el huésped. ${ }^{8-11}$

Recientemente, la enfermedad periodontal ha sido identificada como un factor de riesgo potencial para el bajo peso al nacer y partos prematuros, y podría ser uno de los factores asociados con parte del 50\% de nacimientos de niños prematuros sin factores de riesgo establecidos. Se ha sugerido que esta relación podría originarse por la estimulación de las membranas fetales o la síntesis de las prostaglandinas por medio de citoquinas producidas por tejidos gingivales inflamados o a través del efecto de la endotoxina derivada de la infección periodontal. ${ }^{11-24}$
En los modelos animales se ha demostrado que la endotoxina produce retardo del crecimiento fetal. Por otra parte, los monocitos periféricos obtenidos de las pacientes con enfermedad periodontal mostraban un aumento en la liberación de los mediadores bacterianas inflamatorios tales como el PgE2, la IL- $\beta$ $y$ el TNF- $\alpha$, cuando se enfrenta a endotoxinas bacterianas. ${ }^{18}$ La endotoxina derivada de los patógenos periodontales en las mujeres con enfermedad periodontal puede generar el parto prematuro a través de la activación del macrófago-monocito en la sangre periférica y la decidua. ${ }^{11}$

Cada vez es mayor la evidencia que soporta esta relación; por tal motivo para evitar un proceso inflamatorio oral que evolucione a una periodontitis con posibles repercusiones severas en el embarazo por extensión sistémica, las futuras madres deben recibir atención oral profesional regular con gran énfasis en la higiene a lo largo del embarazo. ${ }^{25-27}$

\section{El concepto de "Transmisibilidad de Strepto- coccus mutans"}

Numerosas investigaciones han demostrado que las madres que presentan infecciones orales pueden ser fuentes potenciales de infección para sus hijos. ${ }^{28-41}$ Durante más de tres décadas, se ha reconocido que la caries dental es una enfermedad infecciosa y transmisible, fuertemente modificada por la dieta. La infancia temprana es la época más importante para la futura salud dental. Durante este periodo los dientes primarios erupcionan, las bacterias colonizan los dientes, y la salud oral en el niño se define. ${ }^{32}$

En la literatura científica se ha comprobado que los microorganismos implicados en la formación de un biofilm metabólicamente asociado a desmineralización del esmalte dental, pueden ser transmitidos entre individuos, pues se han encontrado evidencias de especies con semejanzas genéticas entre madres e hijos. ${ }^{28-33}$

Actualmente se reconoce que la madre usualmente es la fuente primaria de infección de Streptococcus mutans (SM) a su niño y la mala higiene oral materna así como los hábitos en la dieta, aumentan la posibilidad de transmisión. Las madres de niños infectados muestran niveles más elevados de SM, cepillado menos frecuente, niveles más elevados de placa y mayor frecuencias diaria de consumo de pasabocas y exposiciones al azúcar en comparación con madres que no tienen niños infectados. ${ }^{39}$ Los hábitos de crianza también facilitan la transferencia de saliva al niño, como al compartir alimentos y utensilios; la práctica de hábitos que implican el contacto cercano como: amamantamiento y dormir junto a la madre, también ha demostrado una asociación significativa. ${ }^{39}$ 
Por estas razones, el establecimiento y la propagación de SM puede y debe evitarse o retardarse por medio de estrategias dirigidas a la fuente principal de la infección y, de esta manera, prevenir la aparición de caries de la infancia temprana.

\section{La educación de las gestantes como estrategia preventiva}

Está demostrada la influencia de la educación de las madres en la experiencia de caries en los niños. ${ }^{42,43}$ En nuestro medio, la mayoría de las mujeres en embarazo no reciben instrucciones durante esta época relacionadas con la salud oral, aún cuando ésta es una fase en que se deben incentivar las instrucciones y es la oportunidad para introducir programas preventivos. ${ }^{33}$

Las mujeres de bajos ingresos tienen la dificultad de encontrar cuidados dentales para ellas y sus hijos. Las pacientes en embarazo con frecuencia tiene alguna forma de molestia dental y solo un mínimo porcentaje recibe tratamiento. La educación prenatal es clave para el cuidado dental del niño porque las madres deben servir como modelos para ellos y las intervenciones con los niños tienen mucha más probabilidad de éxito en un ambiente donde la madre ya es una paciente exitosa. ${ }^{33}$

Chacón y colaboradores visitaron un total de 16 Empresas Promotoras de Salud (EPS), en la ciudad de Bucaramanga, para indagar sobre la existencia de programas que promocionen la salud oral en las $\mathrm{mu}-$ jeres embarazadas. Algunas entidades empleaban un programa de salud que proporcionaba cierta información, pero no ejecutaban tratamientos integrales y no se reportó su cobertura ni su efectividad. A nivel municipal, se visitó la Secretaría de Salud y el Instituto de Salud de Bucaramanga (ISABU) para indagar sobre la existencia de programas médicos para la mujer embarazada y se observó que no se incluye un anexo de salud bucodental específico para el control de esta población. ${ }^{4}$

Las consecuencias se reflejan en las condiciones de salud oral de las gestantes ya que se observó que el $33 \%$ presentaba periodontitis incipiente, el $47 \%$ periodontitis moderada y el $6 \%$ periodontitis avanzada. $\mathrm{El}$ índice COP presentó un promedio de 14.2 , lo que indica que más de la mitad de los dientes han sido afectados o han sufrido algún proceso patológico. El ICP registró un promedio de 1.82 lo que implica una higiene oral de regular a deficiente. ${ }^{4}$

La mujer embarazada es una paciente muy receptiva a las instrucciones que conlleven a una mejoría de su estado de salud y la de su hijo, por lo que este es un momento crucial para destacar la importancia de las medidas de higiene oral, disminuir así los efectos de los cambios hormonales sobre la encía y evitar un proceso inflamatorio que desarrolle una verdadera periodontitis con repercusiones severas en el resultado del embarazo. ${ }^{4}$ Las futuras madres deben recibir educación en salud oral, para evitar la aparición de esta patología, además de evitar la aparición de caries de la infancia temprana en sus niños por transmisión de cepas de estreptococos de madre a hijo. ${ }^{44}$

El propósito de este estudio fue diseñar, ejecutar y evaluar la efectividad de un programa preventivo en salud oral para controlar los factores de riesgo asociados a caries y enfermedad periodontal en las gestantes afiliadas a la EPS Salud Total Bucaramanga.

\section{MATERIALES Y MÉTODOS}

Se desarrolló un ensayo clínico controlado con el fin de comparar la efectividad de dos programas preventivos en salud oral ejecutados en dos grupos de gestantes asignadas mediante aleatorización por bloques a ser grupo intervenido (20 gestantes) y control (20 gestantes). El universo y la muestra la constituyeron 40 mujeres gestantes que cumplieron con los criterios establecidos. Los criterios de inclusión fueron: maternas con edades entre 15 y 45 años de edad, en su segundo o tercer trimestre de embarazo, afiliadas a la EPS Salud Total Bucaramanga, adheridas al programa de control prenatal, sistémicamente sanas, quienes desearon participar en el estudio y firmaron el consentimiento informado. Los criterios de exclusión fueron: pacientes bajo tratamiento antibiótico, fumadoras, con infecciones del tracto genitourinario, o que planearan cambiar de residencia durante los próximos seis meses.

Se consideraron variables dependientes: índice de creencias saludables, índice de prácticas saludables, dientes sanos, índice COP, necesidad de tratamiento categorizada como ninguno, necesidad de tratamiento obturación de una, dos, tres o más superficies y necesidad de extracción, el índice comunitario periodontal de necesidades de tratamiento (ICPTN), necesidades de tratamiento e índice cuantificado de placa (ICP). Las variables independientes fueron: las sociodemográficas y la variable intervención.

La prueba piloto se efectuó con 10 gestantes que cumplieron con los criterios de inclusión. En ellas se evaluaron los instrumentos, la comprensión de las preguntas, la aceptabilidad y la tasa de respuesta de las gestantes evaluadas. Adicionalmente se evaluaron los mecanismos de aleatorización, los procedimientos de sistematización y las pruebas estadísticas a aplicar. Previamente, una examinadora clínica fue apropiadamente calibrada para la medición respectiva de los índices con la ayuda de una epidemióloga y una periodoncista. 
Los procedimientos se desarrollaron de la siguiente manera:

\section{Demanda inducida}

Las gestantes se vincularon al programa a través del curso psicoprofiláctico, se permitió el ingreso a una de las sesiones con el fin de explicar a las maternas los aspectos relacionados con el estudio e invitarlas a participar.

\section{Primera reunión}

En este primer encuentro, los objetivos y planeación del programa se explicaron en detalle a las pacientes. Las mujeres que decidieron participar, firmaron el consentimiento informado, llenaron el cuestionario para recolección de las variables sociodemográficas, y las encuestas de creencias sobre salud y prácticas de higiene oral. A las mujeres se les brindó un refrigerio y la reunión se dio por terminada cuando todas las gestantes entregaron sus encuestas. Cada una de ellas fue citada a consulta odontológica para la evaluación de su condición de salud oral.

\section{Primera consulta odontológica}

A todas las gestantes se les dio la bienvenida y se les agradeció el hecho de participar en el estudio. Se presentó a la auxiliar e inmediatamente la paciente fue invitada a sentarse en la unidad odontológica donde se le colocó un peto desechable. Se inició con el diagnóstico del estado de la dentición y las necesidades de tratamiento, de acuerdo a lo determinado por el Ensab III. ${ }^{45}$

Para las necesidades de tratamiento se registraron en la casilla correspondiente a cada diente las actividades necesarias para llevar a las pacientes a una adecuada condición de salud bucodental. ${ }^{45} \mathrm{~A}$ las mujeres del grupo experimental, se les dieron las recomendaciones sobre sus necesidades de tratamiento con el fin de promover la demanda del servicio y de esta manera brindar la atención requerida. A las gestantes del grupo control no se les realizaron sugerencias.

Posteriormente, se realizó la evaluación del estado periodontal utilizando el índice comunitario periodontal de necesidades de tratamiento ICPNT. ${ }^{45}$

Finalmente, se evaluó el acumulo de placa bacteriana según el índice cuantificado de placa. ${ }^{46,47}$

En ambos grupos el examen clínico se realizó con una sonda periodontal, y espejo dental en el consultorio odontológico de la IPS, no se incluyó examen radiográfico.

Una vez finalizado el examen, a todas las gestantes del grupo experimental, se les realizó terapia mecánica (profilaxis profesional y detartraje supra y subgin- gival), por parte una higienista oral entrenada. Se reforzaron las instrucciones de higiene oral a través de un cepillado interactivo. Las pacientes se pusieron al tanto del estado de actividad de caries de sus bocas y se les indicaron las necesidades de tratamiento. Ellas recibieron un kit de higiene oral que contenía un cepillo, seda, un tubo de crema dental y un enjuague bucal, junto con un material educativo impreso (folleto). Al finalizar la consulta, se asignó una nueva cita para la conferencia y la presentación del video "Una mirada adulta".

\section{Segunda reunión}

A las gestantes del grupo experimental, se les dictó una conferencia relacionada con los cambios físicos, hormonales, gingivales y en la dieta durante el embarazo; los factores etiológicos de la caries dental, la enfermedad periodontal y cómo esta infección produce efectos adversos en el embarazo. Se dieron instrucciones sobre las medidas preventivas que se encuentran disponibles para evitar la aparición o controlar la entidad. También se tocaron temas relacionados con las creencias sobre salud oral y las prácticas de higiene oral durante el embarazo.

Con el fin de evaluar los conocimientos adquiridos, se les entregó el instrumento para que llenaran nuevamente la encuesta de creencias sobre salud y prácticas de higiene oral. Finalmente se les presentó el video: "Una mirada adulta" y se les entregaron dos folletos de educación en salud oral obsequiados por Colgate ${ }^{\circledR}$.

Las gestantes del grupo control recibieron el programa ejecutado por la EPS y posteriormente diligenciaron la encuesta.

\section{Segunda cita odontológica}

Para evaluar el impacto del programa al finalizar las actividades, se realizó nuevamente en ambos grupos:

- Evaluación del estado de la dentición y las necesidades de tratamiento. ${ }^{45}$

- Evaluación del estado periodontal. ${ }^{45}$

- Evaluación del acumulo de placa bacteriana según el índice cuantificado de placa. ${ }^{46,47}$

El plan de análisis estadístico incluyó el calculo de medidas de resumen según la naturaleza de la variable; para el análisis bivariado se consideró como variable de salida tipo de intervención y las otras variables como explicatorios, para ello se aplicaron test de $\mathrm{Chi}^{2}$ cuadrado y test Exacto de Fisher y para las cuantitativas prueba $t$ de Student o test de rangos de Wilcoxon. Para todo el análisis se considero un nivel de significancia de alfa $\square=0.05$.

La presente investigación se acogió a la reglamentación establecida en la resolución 008430 de 1993, par- 
ticularmente con los apartes relacionados con el trabajo con gestantes; recibió la aprobación del Comité de Ética Institucional, se preservaron los principios de beneficencia, autonomía, confidencialidad y equidad, de acuerdo a lo establecido en la citada resolución.

\section{RESULTADOS}

\section{Análisis univariado}

\section{Factores sociodemográficos y otros relaciona- dos con el embarazo}

En total se estudiaron 40 maternas afiliadas a Salud -Total EPS en la ciudad de Bucaramanga, con una edad promedio de 25.33 $\pm 5,6$ años; el 55\% (22) había nacido en Bucaramanga y 92,5\% (37) procedía de la zona urbana.

La mayor frecuencia de estado civil fue el de casada en un $42,5 \%$ (17), seguido de un $40 \%$ (16) que vivía en unión libre. El 47,5\% (19) era de un estrato socioeconómico medio bajo, y el 75\% (30) de las gestantes había terminado su bachillerato. El 52,5\% (21) trabajaba fuera del hogar. Sobresale un $50 \%$ (20) de embarazos planeados, y una mediana gestacional de $25,7 \pm 3,9$ semanas. El $72,5 \%$ (29) de todas las mujeres eran madres primerizas (Tabla 1).

Tabla 1. Descripción de variables sociodemográficas y las relacionadas con el embarazo en las gestantes afiliadas a una EPS en Bucaramanga analizadas en forma global y según intervención.

\begin{tabular}{|c|c|c|c|c|}
\hline VARIABLE & $\begin{array}{c}\text { Global } \\
n=40 \\
\text { Frec. }(\%)\end{array}$ & $\begin{array}{c}\text { Intervenidas } \\
n=20 \\
\text { Frec. }(\%)\end{array}$ & $\begin{array}{c}\text { No intervenidas } \\
\mathrm{n}=20 \\
\text { Frec. (\%) }\end{array}$ & $\mathbf{p}^{*}$ \\
\hline Población & $40(100 \%)$ & $20(50 \%)$ & $20(50 \%)$ & \\
\hline \multicolumn{5}{|l|}{ Edad } \\
\hline 15 a 25 & $24(60 \%)$ & $11(55 \%)$ & $13(65 \%)$ & 0,827 \\
\hline 26 a 30 & $9(22,5 \%)$ & $5(25 \%)$ & $4(20 \%)$ & \\
\hline Más de 30 & $7(17,5 \%)$ & $4(20 \%)$ & $3(15 \%)$ & \\
\hline \multicolumn{5}{|l|}{ Lugar de Nacimiento } \\
\hline Otro & $18(45 \%)$ & $10(55,56 \%)$ & $8(44,44 \%)$ & $0,525^{* *}$ \\
\hline Bucaramanga & $22(55 \%)$ & $10(45,45 \%)$ & $12(54,55 \%)$ & \\
\hline \multicolumn{5}{|l|}{ Procedencia } \\
\hline Zona Rural & $3(7,5 \%)$ & $1(33,33 \%)$ & $2(66,67 \%)$ & $1^{*}$ \\
\hline Zona Urbana & $37(92,5 \%)$ & $19(51,35 \%)$ & $18(48,65 \%)$ & \\
\hline \multicolumn{5}{|l|}{ Estado Civil } \\
\hline Casada & $17(42,5 \%)$ & $10(58,82 \%)$ & $7(41,18 \%)$ & $0,520^{*}$ \\
\hline Soltera & $6(15 \%)$ & $2(33,33 \%)$ & $4(66,67 \%)$ & \\
\hline Unión Libre & $16(40 \%)$ & $7(43,75 \%)$ & $9(56,25 \%)$ & \\
\hline Viuda & $1(2,5 \%)$ & $1(100 \%)$ & & \\
\hline \multicolumn{5}{|l|}{ Estrato Socioeconómico } \\
\hline 1 Bajo-Bajo & $1(2,5 \%)$ & -- & $1(100 \%)$ & $0,472^{* *}$ \\
\hline 2 Bajo & $15(37,5 \%)$ & $8(53,33 \%)$ & $7(46,67 \%)$ & \\
\hline 3 Medio-Bajo & $19(47,5 \%)$ & $8(42,11 \%)$ & $11(57,89 \%)$ & \\
\hline 4 Medio & $4(10 \%)$ & $3(75 \%)$ & $1(25 \%)$ & \\
\hline 5 Medio-Alto & $1(2,5 \%)$ & $1(100 \%)$ & - & \\
\hline \multicolumn{5}{|l|}{ Escolaridad } \\
\hline Primaria & $3(7,5 \%)$ & $1(33,33 \%)$ & $2(66,67 \%)$ & $0,469^{* *}$ \\
\hline Secundaria & 30 (75\%) & $17(56,67 \%)$ & $13(43,33 \%)$ & \\
\hline Pregrado & $6(15 \%)$ & $2(33,33 \%)$ & $4(66,67 \%)$ & \\
\hline Postgrado & $1(2,5 \%)$ & -- & $1(100 \%)$ & \\
\hline \multicolumn{5}{|l|}{ Ocupación } \\
\hline Trabaja fuera de casa & $21(52,5 \%)$ & $8(38,10 \%)$ & $13(61,90 \%)$ & $0,113^{* *}$ \\
\hline Hogar & $19(47,5 \%)$ & $12(63,16 \%)$ & $7(36,84 \%)$ & \\
\hline Embarazo deseado & $20(50 \%)$ & $9(45 \%)$ & $11(55 \%)$ & $0,527^{* *}$ \\
\hline Madres primerizas & $29(72,5)$ & $13(45 \%)$ & $16(55,2 \%)$ & $0,288^{* *}$ \\
\hline
\end{tabular}


Índice de creencias e índice prácticas saludables

El índice de creencias saludables fue regular, al inicio del estudio, en el $72,5 \%$ (29) de las gestantes, con un promedio de $18,9 \pm 3,2$ preguntas respondidas adecuadamente; finalizada la intervención el índice de creencias saludables fue bueno en el 65\% (26) de las mujeres, con un pro- medio de 22,6 $\pm 1,4$ preguntas respondidas acertadamente. Lo mismo se observó para el índice de prácticas saludables, al principio de estudio fue regular para el 55\% (22) de ellas, con un promedio de 23,5 \pm 4 de preguntas acertadas, finalizada la intervención el índice fue bueno en el $72,5 \%$ (29) de las gestantes, con un promedio de $28,3 \pm 6$ preguntas respondidas adecuadamente. Los hallazgos se encuentran en la tabla 2 .

Tabla 2. Descripción de las creencias y prácticas saludables de las gestantes afiliadas a una EPS, en Bucaramanga analizadas en forma global, antes y después de la intervención.

\begin{tabular}{|c|c|c|c|c|c|c|c|c|}
\hline \multirow{3}{*}{ VARIABLE } & \multirow{3}{*}{$\begin{array}{c}\text { X } \pm \text { D.E } \\
\text { Global } \\
\text { Antes }\end{array}$} & \multirow{2}{*}{\multicolumn{2}{|c|}{$\begin{array}{c}\text { X } \pm \text { D.E } \\
\text { Antes }\end{array}$}} & \multirow{3}{*}{$\mathbf{p}^{*}$} & \multirow{3}{*}{$\begin{array}{c}\text { X } \pm \text { D.E } \\
\text { Global } \\
\text { Después }\end{array}$} & \multirow{2}{*}{\multicolumn{2}{|c|}{$\begin{array}{l}\mathrm{X} \pm \text { D.E } \\
\text { Después }\end{array}$}} & \multirow{3}{*}{$\mathbf{p}^{*}$} \\
\hline & & & & & & & & \\
\hline & & Intervenidas & $\begin{array}{c}\text { No } \\
\text { intervenidas }\end{array}$ & & & Intervenidas & $\begin{array}{c}\text { No } \\
\text { intervenidas }\end{array}$ & \\
\hline \multicolumn{9}{|l|}{$\begin{array}{l}\text { Índice de creencias } \\
\text { saludables }\end{array}$} \\
\hline $\begin{array}{l}\text { Analizadas en forma } \\
\text { cuantitativa }\end{array}$ & $18,9 \pm 3,2$ & $18,6 \pm 3,7$ & $19,2 \pm 2,7$ & 0,5292 & $22,6 \pm 3,9$ & $26 \pm 1,4$ & $19,35 \pm 2,6$ & $<0,0001^{* * *}$ \\
\hline \multicolumn{9}{|l|}{$\begin{array}{l}\text { Índice de prácticas } \\
\text { saludables }\end{array}$} \\
\hline $\begin{array}{l}\text { Analizadas en forma } \\
\text { cuantitativa }\end{array}$ & $23,5 \pm 4$ & $24 \pm 4$ & $23 \pm 4,2$ & 0,5907 & $28,3 \pm 6$ & $33,35 \pm 2$ & $23,2 \pm 4,2$ & $0,001^{* * *}$ \\
\hline \multicolumn{9}{|l|}{$\begin{array}{l}\text { Índice de creencias } \\
\text { saludables }\end{array}$} \\
\hline \multicolumn{9}{|l|}{$\begin{array}{l}\text { Analizadas en forma } \\
\text { cualitativa }\end{array}$} \\
\hline Bueno & $11(27,50 \%)$ & $5(45,45 \%)$ & $6(54,55 \%)$ & 0,723 & $26(65 \%)$ & $20(77 \%)$ & $6(23,08 \%)$ & $<0,0001^{* *}$ \\
\hline Regular & $29(72,50 \%)$ & $15(52 \%)$ & $14(48,28 \%)$ & & $14(35 \%)$ & ----- & $14(100 \%)$ & \\
\hline \multicolumn{9}{|l|}{$\begin{array}{l}\text { Índice de prácticas } \\
\text { saludables }\end{array}$} \\
\hline \multicolumn{9}{|l|}{$\begin{array}{l}\text { Analizadas en forma } \\
\text { cualitativa }\end{array}$} \\
\hline Bueno & $18(45 \%)$ & $9(50 \%)$ & $9(50 \%)$ & 1 & $29(72,5 \%)$ & $20(69 \%)$ & $9(31,03 \%)$ & $<0,0001^{* *}$ \\
\hline Regular & $22(55 \%)$ & $11(50 \%)$ & $11(50 \%)$ & & $11(27,5 \%)$ & ----- & $11(100 \%)$ & \\
\hline
\end{tabular}

** Chi cuadrado ${ }^{* * *} \mathrm{t}$ de Student $\alpha=0.05$

\section{Estado de la dentición y necesidades de tra- tamiento}

Al inicio de estudio el índice COP presentó un promedio de $9,4 \pm 6,1$, el componente de obturados es que más le aporta a este índice con una media de $6,7 \pm 4,6$ obturados. Después de la intervención los criterios anteriormente mencio- nados registraron un comportamiento similar. Es importante anotar que antes y después de la intervención las gestantes registraron un promedio de 18 6,6 dientes sanos (Tabla 3). El promedio de dientes sin necesidad de tratamiento antes de la intervención fue de $26,62 \pm 2,6$ y culminada la intervención los resultados son muy similares (Tabla 4). 
Tabla 3. Descripción de las condiciones de salud oral de las gestantes afiliadas a una EPS, en Bucaramanga analizadas en forma global antes y después de la intervención.

\begin{tabular}{|c|c|c|c|c|c|c|c|c|}
\hline \multirow{3}{*}{ VARIABLE } & \multirow{3}{*}{$\begin{array}{l}\text { X } \pm \text { D.E } \\
\text { Global } \\
\text { Antes }\end{array}$} & \multicolumn{2}{|c|}{$\mathrm{X} \pm$ D.E } & \multirow{3}{*}{$\mathbf{p}^{*}$} & \multirow{3}{*}{$\begin{array}{l}\text { X } \pm \text { D.E } \\
\text { Global } \\
\text { Después }\end{array}$} & \multicolumn{2}{|c|}{ X \pm D.E } & \multirow{3}{*}{$\mathrm{p}^{*}$} \\
\hline & & \multicolumn{2}{|c|}{ Antes } & & & \multicolumn{2}{|c|}{ Después } & \\
\hline & & Intervenidas & No intervenidas & & & Intervenidas & No intervenidas & \\
\hline Sanos & $18 \pm 6,6$ & $18,6 \pm 6,2$ & $17,25 \pm 7,07$ & 0,7759 & $18 \pm 6,6$ & $18,6 \pm 6,2$ & $17,25 \pm 7,07$ & $0,7759^{*}$ \\
\hline Cariados & $1,2 \pm 2,37$ & $1,15 \pm 2$ & $1,25 \pm 3$ & 0,878 & $1.02 \pm 2,18$ & $8 \pm 1,2$ & $1,25 \pm 3$ & $0,9022^{*}$ \\
\hline Obturados & $6,7 \pm 4,61$ & $5,95 \pm 4,7$ & $7,45 \pm 4,52$ & 0,3107 & $6,7 \pm 4,74$ & $6 \pm 4,5$ & $7,45 \pm 4,52$ & $0,3074^{* * *}$ \\
\hline Perdidos & $1,4 \pm 1,70$ & $1,45 \pm 1,66$ & $1,4 \pm 1,8$ & 0,7246 & $1,42 \pm 1,70$ & $1,45 \pm 1,66$ & $1,4 \pm 1,8$ & $0,7246^{*}$ \\
\hline $\mathrm{COP}$ & $9,4 \pm 6,12$ & $8,75 \pm 6$ & $10 \pm 6,38$ & 0,5093 & $9,3 \pm 6,16$ & $8,55 \pm 6$ & $10 \pm 6,38$ & $0,4488^{* * *}$ \\
\hline
\end{tabular}

${ }^{*}$ Test de rangos de Wilconson ${ }^{* * *}$ t de Student $\alpha=0.05$

Tabla 4. Descripción de las necesidades de tratamiento de las gestantes afiliadas a una EPS, en Bucaramanga analizadas en forma global antes y después de la intervención.

\begin{tabular}{|c|c|c|c|c|c|c|c|c|}
\hline \multirow{3}{*}{ VARIABLE } & \multirow{3}{*}{$\begin{array}{l}\text { X } \pm \text { D.E } \\
\text { Global } \\
\text { Antes }\end{array}$} & \multicolumn{2}{|c|}{ X \pm D.E } & \multirow{3}{*}{$\mathbf{p}^{*}$} & \multirow{3}{*}{$\begin{array}{l}\text { X } \pm \text { D.E } \\
\text { Global } \\
\text { Después }\end{array}$} & \multicolumn{2}{|c|}{$\mathrm{X} \pm$ D.E } & \multirow{3}{*}{$\mathrm{p}^{*}$} \\
\hline & & \multicolumn{2}{|c|}{ Antes } & & & \multicolumn{2}{|c|}{ Después } & \\
\hline & & Intervenidas & $\begin{array}{c}\text { No } \\
\text { intervenidas } \\
\end{array}$ & & & Intervenidas & $\begin{array}{c}\text { No } \\
\text { intervenidas } \\
\end{array}$ & \\
\hline Ninguno & $26,62 \pm 2,6$ & $26,8 \pm 1,81$ & $26 \pm 1,4$ & 0,8322 & $26,8 \pm 2,44$ & $27,2 \pm 1,19$ & $26,4 \pm 2,23$ & $0,8322^{*}$ \\
\hline $\begin{array}{l}\text { Obturación de } \\
\text { una superficie }\end{array}$ & $0,87 \pm 2,15$ & $0,85 \pm 1,46$ & $0,9 \pm 2,71$ & 0,4617 & $0,75 \pm 2,04$ & $0,76 \pm 1,09$ & $0,9 \pm 2,71$ & $0,7048^{*}$ \\
\hline $\begin{array}{l}\text { Obturación de } \\
\text { dos superficies }\end{array}$ & $0,75 \pm 0,59$ & $0,1 \pm 0,44$ & $0,25 \pm 0,71$ & 0,311 & $0,15 \pm 0,53$ & $0,05 \pm 0,22$ & $0,25 \pm 0,71$ & $0,2865^{*}$ \\
\hline $\begin{array}{l}\text { Obturación de } \\
\text { tres superficies }\end{array}$ & $0,12 \pm 0,40$ & $0,2 \pm 0,52$ & $0,05 \pm 0,22$ & 0,2865 & $0,1 \pm 0,37$ & $0,15 \pm 0,48$ & $0,05 \pm 0,22$ & $0,534^{*}$ \\
\hline
\end{tabular}

${ }^{*}$ Test de rangos de Wilconson $\alpha=0.05$

\section{Estado de salud periodontal y necesidades de tratamiento}

El 52,5\% (21) de las gestantes presentaron cálculos, el 32,5\% (13) bolsas de 4 a $5 \mathrm{~mm}$, y el 15\% (6) sangrado al sondaje. Por lo anterior el $85 \%$ (34) requerían detartraje, profilaxis e instrucciones de higiene oral, y el 15\% (6) solo instrucciones de hi- giene oral. Luego de la intervención, el 35\% (14) de las gestantes presentaron bolsas de 4 a $5 \mathrm{~mm}$, $27,5 \%$ (11) un periodonto sano, el 27,5\% (11) cálculos, y el $10 \%$ (4) sangrado al sondaje. De esta manera, el 62,5\% (25) requerían detartraje, profilaxis e instrucciones de higiene oral, el 27,5\% (11) no requería tratamiento y el $10 \%$ (4) requería sólo instrucciones de higiene oral (Tabla 5). 
Tabla 5. Descripción del estado de salud periodontal y las necesidades de tratamiento de las gestantes afiliadas a una EPS, en Bucaramanga analizadas en forma global antes y después de la intervención.

\begin{tabular}{|c|c|c|c|c|c|c|c|c|}
\hline \multirow{2}{*}{ VARIABLE } & \multirow{2}{*}{$\begin{array}{c}\begin{array}{c}\text { Global } \\
\mathrm{n}=\mathbf{4 0}\end{array} \\
\text { Frec. Antes }\end{array}$} & \multicolumn{2}{|c|}{$\begin{array}{c}\text { Frecuencia } \\
\text { Antes }\end{array}$} & \multirow{2}{*}{$\mathbf{p}^{*}$} & \multirow{2}{*}{$\begin{array}{c}\begin{array}{c}\text { Global } \\
\mathbf{n}=\mathbf{4 0}\end{array} \\
\text { Frec. Desp }\end{array}$} & \multicolumn{2}{|c|}{$\begin{array}{l}\text { Frecuencia } \\
\text { Después }\end{array}$} & \multirow{2}{*}{$\mathbf{p}^{*}$} \\
\hline & & Intervenidas & $\begin{array}{c}\text { No } \\
\text { intervenidas }\end{array}$ & & & Intervenidas & $\begin{array}{c}\text { No } \\
\text { intervenidas }\end{array}$ & \\
\hline \multicolumn{9}{|l|}{$\begin{array}{l}\text { Índice periodontal } \\
\text { comunitario }\end{array}$} \\
\hline Normal & ------ & ------ & ------ & 0,693 & $11(27,5 \%)$ & $11(100 \%)$ & ------ & $<0,0001^{*}$ \\
\hline Sangrado & $6(15 \%)$ & $4(66,7 \%)$ & $2(33,3 \%)$ & & $4(10 \%)$ & $2(50 \%)$ & $2(50 \%)$ & \\
\hline Cálculo & $21(52,5 \%)$ & $9(42,9 \%)$ & $12(57,1 \%)$ & & $11(27,5 \%)$ & ----- & $11(100 \%)$ & \\
\hline Bolsas de 4 a $5 \mathrm{~mm}$ & $13(32,5 \%)$ & $7(53,9 \%)$ & $6(46,1 \%)$ & & $14(35 \%)$ & $7(50 \%)$ & $7(50 \%)$ & \\
\hline Bolsas de $6 \mathrm{~mm}$ & ----- & ----- & ---- & & ---- & ---- & ---- & \\
\hline \multicolumn{9}{|l|}{$\begin{array}{l}\text { Necesidades de Trata- } \\
\text { miento }\end{array}$} \\
\hline No requiere & ----- & ----- & ----- & 0,661 & $11(27,5 \%)$ & $11(100 \%)$ & ----- & $<0,0001^{*}$ \\
\hline $\begin{array}{l}\text { Instrucciones de } \\
\text { higiene oral } \\
\text { Detartraje, profilaxis }\end{array}$ & $6(15 \%)$ & $4(66,7 \%)$ & $2(33,3 \%)$ & & $4(10 \%)$ & $2(50 \%)$ & $2(50 \%)$ & \\
\hline $\begin{array}{l}\text { e instrucciones de } \\
\text { higiene oral } \\
\text { Detartraje, profilaxis }\end{array}$ & $34(85 \%)$ & $16(47,1 \%)$ & $18(52,9 \%)$ & & $25(62,5 \%)$ & $7(28 \%)$ & $18(72 \%)$ & \\
\hline $\begin{array}{l}\text { e instrucciones de } \\
\text { higiene oral } \\
\text { Más tratamiento } \\
\text { complejo }\end{array}$ & ----- & ---- & ------ & & ---- & ----- & ---- & \\
\hline
\end{tabular}

*Exacto de Fisher $\alpha=0,05$

\section{Índice cuantificado de placa}

El índice fue regular para el 45\% (18) de las gestantes, bueno para el 35\% (14) y malo para el 20\%
(8) de ellas, antes de la intervención. Posteriormente, fue bueno para el $80 \%$ (32) de las maternas, regular para el 15\% (5) (Tabla 6).

Tabla 6. Descripción del índice cuantificado de placa de las gestantes afiliadas a Salud Total EPS, Bucaramanga analizadas en forma global, antes y después de la intervención.

\begin{tabular}{|c|c|c|c|c|c|c|c|c|}
\hline \multirow[b]{2}{*}{ VARIABLE } & \multirow{2}{*}{$\begin{array}{c}\text { X } \pm \text { D.E } \\
\text { Global } \\
\text { Antes }\end{array}$} & \multicolumn{2}{|c|}{$\begin{array}{c}\text { X } \pm \text { D.E } \\
\text { Antes }\end{array}$} & \multirow[b]{2}{*}{$\mathbf{p}^{*}$} & \multirow{2}{*}{$\begin{array}{c}\text { X } \pm \text { D.E } \\
\text { Global } \\
\text { Después }\end{array}$} & \multicolumn{2}{|c|}{$\begin{array}{l}\mathrm{X} \pm \mathrm{D} . \mathrm{E} \\
\text { Después }\end{array}$} & \multirow[b]{2}{*}{$\mathbf{p}^{*}$} \\
\hline & & Intervenidas & $\begin{array}{c}\text { No } \\
\text { intervenidas }\end{array}$ & & & Intervenidas & $\begin{array}{c}\text { No } \\
\text { intervenidas }\end{array}$ & \\
\hline $\begin{array}{l}\text { Índice cuantificado } \\
\text { de placa }\end{array}$ & & & & & & & & \\
\hline $\begin{array}{l}\text { Analizados en forma } \\
\text { cuantitativa } \\
\text { Indice cuantificado } \\
\text { de placa }\end{array}$ & $1,29 \pm, 64$ & $1,54 \pm 0,65$ & $1,04 \pm 0,53$ & 0,0038 & $22,6 \pm 3,9$ & $0,18 \pm 0,11$ & $1,04 \pm 0,53$ & $<0,0001^{*}$ \\
\hline $\begin{array}{l}\text { Analizadas en forma } \\
\text { cualitativa }\end{array}$ & & & & & & & & \\
\hline Bueno & $14(35 \%)$ & $2(14,3 \%)$ & $12(85,7 \%)$ & 0,004 & $32(80 \%)$ & $20(62,5 \%)$ & $12(37,5 \%)$ & $0,004^{* *}$ \\
\hline Regular & $18(45 \%)$ & $12(66,7 \%)$ & $6(33,33 \%)$ & & $6(15 \%)$ & & $6(100 \%)$ & \\
\hline Malo & $8(20 \%)$ & $6(66,75 \%)$ & $2(25 \%)$ & & $2(5 \%)$ & & $2(100 \%)$ & \\
\hline
\end{tabular}

${ }^{*}$ Test de rangos de Wilcoxon ${ }^{* *}$ Exacto de Fisher $\alpha=0,05$ 


\section{Análisis bivariado}

\section{Factores sociodemográficos y otros relaciona- dos con el embarazo}

El análisis bivariado permite observar que para estas variables no se observaron asociaciones estadísticamente significativas, lo que parece ilustrar que los grupos eran similares en cuanto a estas características en la línea de base.

\section{Índice de creencias e índice prácticas saluda- bles}

Para el índice de creencias saludables no se observaron diferencias estadísticamente significativas entre los grupos antes de la intervención $(\mathrm{p}=0.5292)$, fenómeno que si se observó después de la intervención ( $p<0,0001)$, Para esto último es importante destacar que, mientras para el grupo no intervenido la media en el índice de creencias saludables fue $19,35 \pm 2,6$ preguntas que se respondieron acertadamente en el grupo intervenido fue $26 \pm 1,4$.

Un hecho similar se evidenció con el índice de prácticas saludables, con un nivel de significancia después de la intervención de $\mathrm{p}=0,001$; cuyos promedios en el grupo control fue de 23,2 $\pm 4,2$ aciertos vs 33,4 \pm 2 del grupo intervenido (Tabla 2).

\section{Estado de salud periodontal y necesidades de tratamiento}

El estado periodontal y las necesidades de tratamiento tanto para las personas asignadas al grupo intervención como al control mostraron ser muy similares antes de la aplicación del programa $(\mathrm{p}=$ 0.693 y 0.661 , respectivamente). Posterior a este, para estos dos aspectos, se evidenciaron asociaciones estadísticamente significativas con $\mathrm{p}<0.0001$. Se destaca que el $100 \%$ de las personas con periodonto normal habían sido intervenidas, mientras que ninguna del grupo control registró esta condición. En cuanto a necesidad de tratamiento es importante resaltar que del total de personas que requerían detartraje, profilaxis e instrucciones de higiene oral, el $28 \%$ pertenecían al grupo intervenido y el $72 \%$ al grupo control (Tabla 5).

\section{Índice Cuantificado de Placa}

Mostró que antes de la implementación del programa el $75 \%$ de las gestantes intervenidas tenían mala higiene oral y el $25 \%$ de las no intervenidas registraban igual condición $(\mathrm{p}=0.004)$. Después de la aplicación del programa el $62.5 \%$ de las intervenidas y $32.5 \%$ de las no intervenidas tenían buena higiene oral $(p=0.004)$ (Tabla 6$)$.

\section{DISCUSIÓN}

Es fundamental brindar una adecuada atención odontológica a la mujer en etapa de gestación. Brindar una orientación adecuada requiere del conocimiento del odontopediatra acerca de cómo unas creencias y prácticas inadecuadas, pueden afectar no solo la salud de la madre sino también la del bebé.

En este estudio, la mayoría de las gestantes eran casadas 42,5\%; habían terminado su bachillerato; trabajaban fuera de casa $52,5 \%$, eran madres primerizas $72,5 \%$; la mitad de ellas habían programado su embarazo y eran mujeres jóvenes de 25,33 años en promedio. Estos factores han sido reportados en la literatura como protectores para la aparición de caries en las madres y sus hijos. 4,32,33,38-40,42,48

Es bien sabido que la caries y la enfermedad periodontal son entidades que se relacionan con el nivel de educación y los hábitos, por lo que la promoción y la prevención pueden contribuir a una mejor calidad de vida de las pacientes. ${ }^{4,21,33,38,39,48,49}$ En la población estudiada no se observaron diferencias significativas al inicio del ensayo entre los dos grupos, en lo que se refiere a creencias y prácticas saludables, lo que parece indicar que eran grupos homogéneos; sin embargo, al finalizar el estudio se observaron diferencias estadísticamente significativas entre los grupos ( $p<0,0001)$, lo que puede sugerir que estas diferencias fueron producto de la intervención.

Las madres respondieron positivamente a la información brindada, todas ellas se encontraban en control prenatal, estaban afiliadas al sistema de seguridad social en salud, tenían un nivel educativo similar, pertenecían a un estrato socio económico muy relacionado, pero sólo en las que recibieron la intervención se observó una mejoría estadísticamente significativa $(p<0,0001)$. Se puede pensar entonces, que estos elementos no son suficientes para garantizar una buena salud oral, sino que es necesaria la implementación de un programa preventivo enfocado a las gestantes, que se base en la educación, y brinde las herramientas necesarias para que la mujer mejore su condición de salud oral.

De la misma manera, cabe recalcar la importancia del trabajo interdisciplinario que se debe realizar con el equipo que hace parte del programa de control prenatal en la I.P.S, para establecer un buen canal de comunicación entre los diferentes profesionales, ya que por lo general se ignora la importancia del control odontológico durante la gestación y la inocuidad de muchos de los procedimientos que se realizan. ${ }^{3,21,48}$ 
Todas las mujeres del grupo intervenido aceptaron recibir la atención odontológica, sin embargo las necesidades de tratamiento no fueron suplidas, como sucedió en otros estudios en los cuales las gestantes recibieron todas las intervenciones requeridas. ${ }^{11,15,33}$ Infortunadamente, esto podría explicarse porque existe una baja oportunidad de atención en la EPS, y una ausencia del componente educativo; se dispone de poco tiempo para atender a un gran número de pacientes, siendo este un indicador empleado inadecuadamente para medir la eficiencia del profesional. Podría planearse, una evaluación a largo plazo, cuando las gestantes hayan tenido acceso a todos los servicios odontológicos requeridos, debido a que en este estudio la evaluación se realizó al mes de haber hecho el examen clínico. Adicionalmente, los odontólogos manifestaron a las pacientes temor de realizarles procedimientos, especialmente los más invasivos como endodoncias y extracciones.

En este estudio, se evidenció una alta aceptabilidad de las gestantes a este tipo de programas, la gran disposición que tienen para mejorar su estado de salud oral y el interés que manifiestan hacia las verdades científicas que la investigación ha revelado y que son transmitidas por el profesional de la salud, para salir de la ignorancia y romper con los paradigmas que la tradición ha impuesto creando mitos y creencias sin validez que van en detrimento de la salud de las gestantes y de sus hijos. Esto concuerda con lo reportado por Chacón y colaboradores quienes resaltan la alta receptividad de la mujer gestante hacia cualquier medida que implique una mejora en su salud y la de su hijo. ${ }^{4}$

Por otra parte, ninguna de las madres examinadas se encontró libre de enfermedad periodontal, a diferencia de lo reportado por Chacón y colaboradores, quienes en su estudio encontraron un 2,6\% de mujeres con periodonto sano. ${ }^{13} \mathrm{El} 65 \%$ (26) de las mujeres presentaron gingivitis, de ese porcentaje, en el 52\% (21) se hallaron cálculos supra o subgingivales, el $32 \%$ presentó periodontitis moderada, y el $2,5 \%$ periodontitis avanzada. Los resultados difieren con los de Chacón y colaboradores quienes hallaron menor prevalencia de cálculos $32,89 \%$, mayor prevalencia de periodontitis moderada $46,98 \%$, y mayor prevalencia de periodontitis avanzada $6,4 \% .{ }^{4}$

Todas las mujeres del grupo intervenido fueron tratadas antes de parto. Al final del estudio, al $85 \%$ de las mujeres de este grupo se les diagnosticó periodonto sano, mientras que en el grupo no intervenido no se hallaron diferencias entre las dos evaluaciones. Teniendo en cuenta que al inicio del estudio, los grupos eran semejantes, se puede su- poner que las diferencias surgidas entre los grupos son producto de la intervención, tal como lo sugiere la literatura. ${ }^{11,15,33}$

En el índice cuantificado de placa también se observó una diferencia estadísticamente significativa $(p<0,0001)$ entre los grupos, donde el $60 \%$ del grupo intervenido mostró un índice regular al principio del estudio, pero al finalizar se observó que el $100 \%$ de las gestantes presentaban un índice gingival bueno. El grupo no intervenido no mostró cambios. Se podría mencionar que aunque el embarazo es un factor de riesgo para la aparición de gingivitis debido a los cambios hormonales que se presentan, una adecuada higiene oral puede ayudar a prevenir o reducir la severidad de estos cambios inflamatorios tal como se afirma en la literatura. ${ }^{3,5}$

Aunque en la legislación colombiana se establece una atención especial para la mujer gestante, $\mathrm{y}$ aunque se reconoce a este grupo poblacional como vulnerable, es necesario reconocer por parte de los profesionales de la salud, el papel tan relevante que tienen en la búsqueda del bienestar del binomio madre hijo que inicia aún antes de la gestación.

Hay gran desconocimiento de las implicaciones que una mala salud dental y periodontal pueden tener sobre el producto del embarazo. Los resultados de este estudio, tal como se ha reportado, revelan claramente que este tipo de actividades son altamente costoefectivas y que tienen gran impacto sobre la población. ${ }^{4,11,13,15,18,20}$

Seria de gran valor, para nuestra comunidad, realizar estudios a largo plazo para observar no sólo el efecto sobre la salud periodontal sino también sobre la salud dental y su relación con el parto prematuro y/o el bajo peso al nacer de los hijos de las madres intervenidas y no intervenidas, así como la incidencia de caries de la infancia temprana en los hijos.

\section{CONCLUSIONES}

1. El éxito de un programa odontológico preventivo que pretenda mejorar las condiciones de salud oral de la mujer gestante depende, en gran parte, del talento humano capacitado interdisciplinariamente.

2. La mujer, en etapa de gestación, es una paciente muy receptiva frente a las medidas encaminadas a mejorar su salud oral, especialmente si sabe que ésta puede influir en el bienestar de su hijo.

3. Un programa preventivo que promueva creencias y prácticas saludables en la mujer gestante, 
puede ayudar a prevenir o reducir la severidad de los cambios inflamatorios gingivales mediados hormonalmente.

4. Las EPS pueden implementar programas preventivos enfocados a los grupos poblacionales más vulnerables, especialmente las mujeres gestantes, sin que ello implique mayor inversión y así garantizar la prestación de un servicio integral que se ajuste a las necesidades de esta población.

5. A pesar de que la ley contempla la atención odontológica a la mujer gestante, se requiere un protocolo basado en la literatura científi$\mathrm{ca}$, que estandarice los procesos y le brinde al profesional los conocimientos necesarios para que con seguridad y sin temor realice las actividades, procedimientos e intervenciones que requiere la gestante para mejorar su salud oral y procure el bienestar de su bebé.

\section{AGRADECIMIENTO}

A Salud Total EPS por permitir realizar este trabajo con sus afiliadas y dentro de sus instalaciones en la Unidad de Promoción y Prevención de San Pio, en Bucaramanga.

\section{BIBLIOGRAFÍA}

1. Bjelica A, Kapor-Stanulovic N. Pregnancy as a psychological event. Med Pregl 2004; 57: 144 - 148.

2. Herrera JA. Aplicación de un modelo biopsicosocial para la reducción de la morbilidad y mortalidad materna y perinatal en Colombia. Ministerio de Salud. República de Colombia. 1997.

3. Suresh L, Radfar L. Pregnancy and lactation. Oral Surg Oral Med Oral Pathol 2004; 97: 672 - 681.

4. Chacón AF, Gutiérrez ZA, Montañez J, Pieschacón MP, Camargo DM. Factores asociados a caries y enfermedad periodontal en mujeres gestantes, Bucaramanga 2001. Revista Ustasalud 2002; 1: 33 - 39.

5. Kinnby B, Matsson L, Astedt B. Aggravation of gingival inflammatory symptoms during pregnancy associated with the concentration of plasminogen activator inhibitor type 2 (PAI-2) in gingival fluid. J Periodon Res 1996; 3: 271-277.

6. Chamoschine FF, Cardoso M, Lindares SR, De Oliveira V. Processo de saúde-doencas peridontal genograma. Associação Brasileira de Odontologia - ABO-DF Escola de Aperfeiçoamento Profissional - DF Pós-Graduação em Saúde Coletiva. Brasília, julho de 2003.

7. Rodríguez He, López M. El embarazo: Su relación con la salud bucal. Rev Cubana Estomatol. [en línea]. Mayoago. 2003, vol.40, no.2 [fecha de acceso: febrero 13 de 2005], p.0-0. URL disponible en: http://scielo.sld.cu/scielo. php?script $=$ sci_arttext\&pid $=$ S00347507200300020000 9\&lng $=$ es\&nrm $=$ iso

8. Academy Report. Position Paper Diagnosis of Periodontal Diseases. J Periodontol 2003; 74: 1237 - 1247.
9. Wiebe C, Putnins E. The periodontal disease classification system of the American Academy of Periodontology - An Update. J Can Dent Assoc 2000; 66: 594 - 597.

10. Academy Report. Position Paper. Periodontal diseases of children and adolescents. J Periodontol 2003; 74: 1696 1704.

11. Lopez NL, Smith PC, Gutierrez J. Higher risk of preterm birth and low birth weight in women with periodontal disease. J Dent Res 2002; 81: 58 - 63.

12. Offenbacher S, Katz V, Fertik G. Periodontal infection as a possible risk factor for preterm low birth weight. J Periodontol 1996; 67: 1103 - 1113.

13. Dasanayake AP. Poor periodontal health of the pregnant woman as a risk factor for low birth weight. Ann Periodontol. 1998; 3: 206 - 212.

14. Offenbacher S, Jared HL, O'Reilly PG. Potential pathogenic mechanisms of periodontitis associated pregnancy complications. Ann Periodontol 1998; 3: 233 - 250.

15. Lopez NL, Smith PC, Gutierrez J. Periodontal therapy may reduce the risk of preterm low birth weight in women with periodontal disease: A randomized controlled trial. J Periodontol 2002; 73: 911 - 924.

16. Saméer AM, Ghadeer NM. The Prevalence and relationship between periodontal disease and pre-term low birth weight infants at King Khalid University Hospital in Riyadh, Saudi Arabia. J Contemp Dent Practice 2004; 5: 2.

17. Diaz GL, Castellanos SJ. Lesions of the oral mucosa and periodontal disease behavior in pregnant patients. Med Oral Patol Oral Cir Bucal 2002; 9: 430 - 437.

18. Yeo BK, Lim LP, Paquette DW, Williams RC. Periodontal disease - The emergence of a risk for systemic conditions: pre-term low birth weight. Ann Acad Med Singapore 2005; 34: 111 - 116.

19. Davenport ES, Williams CE, Sterne JA, Murad S, Sivapathasundram V, Curtis MA. Maternal periodontal disease and preterm low birth weight: case-control study. J Dent Res 2002; 81: 313 - 318.

20. Louro MP, Fiori HH, Louro FP, Steibel J, Fiori MR. Periodontal disease in pregnancy and low birth weight. J Pediatr (Rio J) 2001; 77: 23 - 28.

21. Felice P, Pelliccioni GA, Checchi L. Periodontal disease as a risk factor in pregnancy. Minerva Stomatol 2005; 54: $255-264$.

22. Löe $\mathrm{H}$, Silness J. Periodontal disease in pregnancy. I. Prevalence and severity. Acta Odontol Scand 1963; 21: 533 551.

23. Jarjoura K, Devine PC, Perez-Delboy A, Herrera-Abreu M, D'alton M, Papanou PN. Markers of periodontal infection and preterm birth. Am J Obstet Gynecol 2005; 192: $513-519$.

24. Goepfert AR, Jeffcoat MK, Andrews WW, Faye-Petersen O, Cliver SP, Goldenberg RL, Hauth JC. Periodontal disease and upper genital tract inflammation in early spontaneous preterm birth. Obstet Gynecol 2004; 104: $777-783$.

25. Lopez NL, Smith PC, Gutierrez J. Periodontal therapy may reduce the risk of preterm low birth weight in women with periodontal disease: A randomized controlled trial. J Periodontol 2002; 73: 911 - 924.

26. Academy Report. Treatment of plaque-induced gingivitis, chronic periodontitis, and other conditions. J Periodontol 2001; 72:1790 - 1800 . 
27. Academy Report. Position Paper. Guidelines for Periodontal Therapy. J Periodontol 2001; 72: 1624 - 1628.

28. Aaltonen AS, Tenovuo J. Association between motherinfant salivary contacts and caries resistance in children: a cohort study. Pediatr Dent 1994; 16: $110-116$.

29. Lindquist B, Emilson CG. Colonization of Streptococcus mutans and Streptococcus sobrinus genotypes and caries development in children to mothers harboring both species. Caries Res 2004; 38: 95 - 103.

30. Tanner AC, Milgrom PM, Ketn R Jr, Mokeen SA, Page RC, Liao SI Riedy CA, Bruss JB. Similarity of the oral microbiota of pre-school children with that of their caregivers in a population-based study. Oral Microbiol Immunol 2002; 17: $379-387$.

31. Mattos-Graner RO, Li Y, caufiled PW, Duncan M, Smith DJ. Genotypic diversity of mutans streptococci in Brazilian nursery children suggests horizontal transmission. J Clin Microbiol 2001; 39: 2313-2316.

32. Arango M, Baena G. Caries de la infancia temprana y factores de riesgo. Revisión de la literatura. Revista Estomatología 2004. 12: 59 - 65.

33. Zanata RL, Navarro MF, Pereira JC, Franco EB, Lauris $\mathrm{JR}$, Barbosa SH. Effect of caries preventive measures directed to expectant mothers on caries experience in their children. Braz Dent J 2003; 14: 75 -81.

34. Palomari DM, Höfling JF, Pizolitto AC, Rosa EA, Negrini TC, Spolidorio LC. Genetic polymorphism of Streptococcus mutans in Brazilian family members. Brazilian J Microbiol 2003; 34: 213 - 217.

35. Grönroos L, Saarela M, Matto J, Tanner-Salo U, Vuorela A, Alaluusua S. Mutacin production by Streptococcus mutans may promote transmission of bacteria from mother to child. Infect and immun 1998; 2595 - 2600.

36. Berkowitz RJ. Acquisition and transmission of Mutans Streptococci. J Calif Dent Assoc 2003; 31: 135 - 138.

37. Berkowitz RJ, Jordan V. Similarity of bacteriocins of Streptococcus mutans from mother and infant. Arch Oral Biol 1982; 26: 147 - 149

38. Van AKL, Seon WK, Purdie DM, Bird PS, Walsh LJ, Tudehope DI. Oral colonization of Streptococcus mutans in sixmonth-old predentate infants. J Dent Res 2001; 80: 2060 $-2065$.
39. Van AKL, Seon WK, Purdie DM, Bird PS, Walsh L J, Tudehope DI. A longitudinal study of Streptococcus mutans colonization in infants after tooth eruption. J Dent Res 2003; 20: 504 - 508.

40. Kohler B, Brattahall D, Krasse B. Preventive measures in mothers influence the establishment of the bacterium Streptococcus mutans in their infants. Arch Oral Biol 1983; 28: $225-231$.

41. Caufield PW, Cutter GR, Dasanayake AP. Initial acquisition of mutans streptococci by infants: evidence for a discrete window of infectivity. J Dent Res 1993; $72: 37$ - 45.

42. Ekstrand $\mathrm{KR}$, Christiansen $\mathrm{m}$, Qvist V. Influence of different variables on the inter-municipality variation in caries experience in Danish adolescents. Caries Res 2003; 37:130 - 41.

43. El método Nexodent. Un programa no operativo de tratamiento de caries NOCTP. URL disponible en: http:// www.nexodent.dk.

44. Lima KC, Neves AA, Sanchez AL, Valente AG, Marsia JG, Castro RA, Ribeiro AA. Relevância clínica do conceito de transmissibilidade da cárie dental. J Bras Odontopediatr Odontol Bebê 2002; 5: 113 - 118.

45. República de Colombia. Ministerio de Salud. III Estudio Nacional de Salud Bucal - ENSAB III, II Estudio Nacional de factores de riesgo de enfermedades crónicas - ENFREC II, 1999.

46. Greene JC Vermillon JR. The oral hygiene index: a method for classifying oral hygiene status. J Am Dent Assoc 1960; 61: 172.

47. Greene JC, Vermillon JR. The simplified oral hygiene index. J Am Dent Assoc 1964; 68: 7.

48. Ferreira A, Benfatti S, Bausells J. Evaluación del conocimiento de las prácticas de salud bucal en gestantes del municipio de Adamantina -SP. Rev Ibero-am Odontopediatr Odontol Bebe 2004; 75: 66 - 77.

49. Garbero I, Delgado A, De Cardenas B. Salud oral en embarazadas: Conocimientos y actitudes. Acta Odontológica Venezolana 2005; 43: 135 - 140.

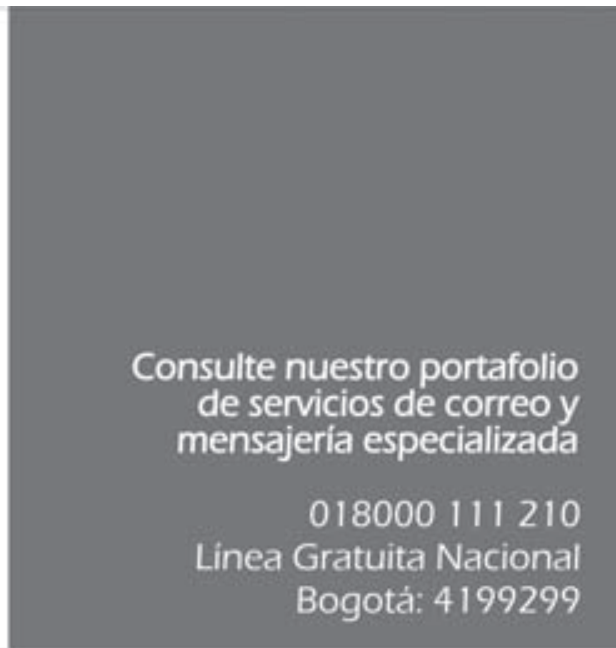

